\title{
Integrating syphilis screening in a large-scale HIV prevention program for key populations: the Avahan experience from India
}

\author{
Mugundu R. Parthasarathy ${ }^{1}$, Prakash Narayanan ${ }^{1}$, Anjana Das ${ }^{1}$, Anup Gurung ${ }^{2}$, \\ Parimi Prabhakar ${ }^{3}$, Teodora $\mathrm{Wi}^{4}$ \\ ${ }^{1}$ FHI360, India Country Office, New Delhi, India \\ ${ }^{2}$ FHI360, Papua New Guinea Country, New Guinea \\ ${ }^{3}$ India HIVIAIDS Alliance, Hyderabad, India \\ ${ }^{4}$ World Health Organization Headquarters, Geneva, Switzerland
}

\begin{abstract}
Introduction: Documented experiences from India on the implementation of syphilis screening in large-scale HIV prevention programs for “key populations at higher risk' (KPs) are limited. Avahan is a large-scale HIV prevention program providing services to more than 300,000 KPs in six high HIV prevalence states of India since 2004. Avahan clinics provide a sexually transmitted infection service package which includes bi-annual syphilis screening. The trends in the coverage of syphilis screening among Avahan clinic attendees were studied retrospectively.

Methodology: Screening was performed using either the Rapid Plasma Reagin (RPR) test or point-of-care immunochromatographic strip test (ICST). Clinic records from 2005 to 2009 were collated in an individual tracking database and analyzed with STATA-10.

Results: Initially the coverage of syphilis screening (2.6\% in 2005) was constrained by the availability and operational complexity of the RPR test. After its introduction in 2007, the use of ICST for screening increased from $7.4 \%$ to $77.0 \%$ and the proportion of clinic attendees screened increased from $9.0 \%$ to $21.6 \%$ during $2007-2009$. The RPR reactivity rates declined from $6.6 \%$ (2006) to 4.4\% (2009).

Conclusion: The data showed improved rates of screening of clinic attendees and declining trends in sero-reactivity over time. The introduction of point-of-care syphilis tests may have contributed to the improved coverage of syphilis screening. The ICST may be considered for initial syphilis screening at other resource-constrained primary care sites in India such as ante-natal clinics and other KP interventions.
\end{abstract}

Key words: key population; syphilis screening; ICST; HIV prevention

J Infect Dev Ctries 2013; 7(6):484-488. doi:10.3855/jidc.2810

(Received 26 June 2012 - Accepted 22 October 2012)

Copyright (C) 2013 Parthasarathy et al. This is an open-access article distributed under the Creative Commons Attribution License, which permits unrestricted use, distribution, and reproduction in any medium, provided the original work is properly cited.

\section{Introduction}

Early detection and treatment of syphilis prevents the adverse medical consequences including continuing transmission to sexual partner/s and increased risk of human immunodeficiency virus (HIV) acquisition. The latent nature of the infection and its painless ulcers are likely to result in reduced treatment-seeking behaviour. Thus promotion of early diagnosis and treatment of sexually transmitted infections (STIs) including syphilis is an essential component of HIV prevention programs [1].

Documented experiences from India on the implementation of syphilis screening in large-scale HIV prevention programs for "key populations at higher risk of HIV exposure" (KPs) are limited. Studies in India over the last ten years have shown syphilis prevalence among female sex workers
(FSWs), men who have sex with men (MSM), and injecting drug users (IDUs), ranging from $1.7 \%$ to $39.7 \%, \quad 3.0 \%$ to $17.0 \%$ and $0.9 \%$ to $19.5 \%$ respectively [2,3].

Avahan is a large-scale HIV prevention program for key populations including female sex workers, men who have sex with men, and injecting drug users, providing services since 2004 in 83 out of 130 districts in six high HIV prevalence states in India. Seven state level implementing partners work through 134 local non-governmental organizations and over 7,000 peer educators and outreach workers [4]. Avahan provides services to more than 220,000 FSWs, 80,000 high-risk MSM and transgendered people, and 18,000 IDUs [5]. The main components of the intervention are peer-led outreach, STI clinical services, community mobilization, and structural interventions. 
A centralized STI capacity building team developed the Clinic Operational Guidelines and Standards, and provided training and supportive supervision for the state lead partners, who in turn provided training and support to the staff of the implementing non-governmental organizations. Biannual syphilis screening was a component of the STI Service Package for the key populations. The STI service package also included syndromic management of STIs, quarterly clinical screening (including speculum and proctoscopic examination), presumptive treatment of asymptomatic gonorrhoea and chlamydia (Cefixime $400 \mathrm{mg}$ and Azithromycin 1g), and risk reduction counselling [6].

A retrospective analysis of Avahan clinic data from the years 2005 to 2009 was conducted to study the trends in the coverage of syphilis screening and sero-reactivity.

\section{Methodology}

Syphilis screening and management was rolled out using standardized guidelines and algorithms as follows. Briefly, the key populations were motivated for syphilis screening through outreach messages and community consultations. The initial strategy was either on-site or off-site screening with nontreponemal tests such as Rapid Plasma Reagin (RPR) with confirmation of reactive results by treponemal tests at an off-site laboratory. However, the operational complexities, particularly for off-site testing, long waiting period for results, fear of venipuncture by key populations, and limited availability of RPR tests at some geographical locations resulted in low uptake of syphilis screening and losses in follow-up. Hence the immunochromatographic strip test (ICST), a point-ofcare rapid syphilis test was introduced in 2007 for use at sites where RPR testing was not available or feasible. Screening algorithms for these sites were revised including ICST as the primary screening test (Figure).

ICST uses whole blood obtained by finger prick. It can be performed by any trained health care worker and stored at room temperature. It also does not require special equipment and results are available in 15 minutes. Treatment was initiated at the same visit for ICST-positive clients who gave a history of either none or incomplete treatment for syphilis. They were encouraged to get RPR tests for baseline and prognostic titers, and for future screening. The baseline RPR result was considered in deciding further treatment for syphilis.

The key populations attending the Avahan clinics were assigned a unique identification number on registration. The clinical records of the KPs attending Avahan clinics from 2005 to 2009 were collated into

Figure 1. Syphilis screening algorithm using ICST

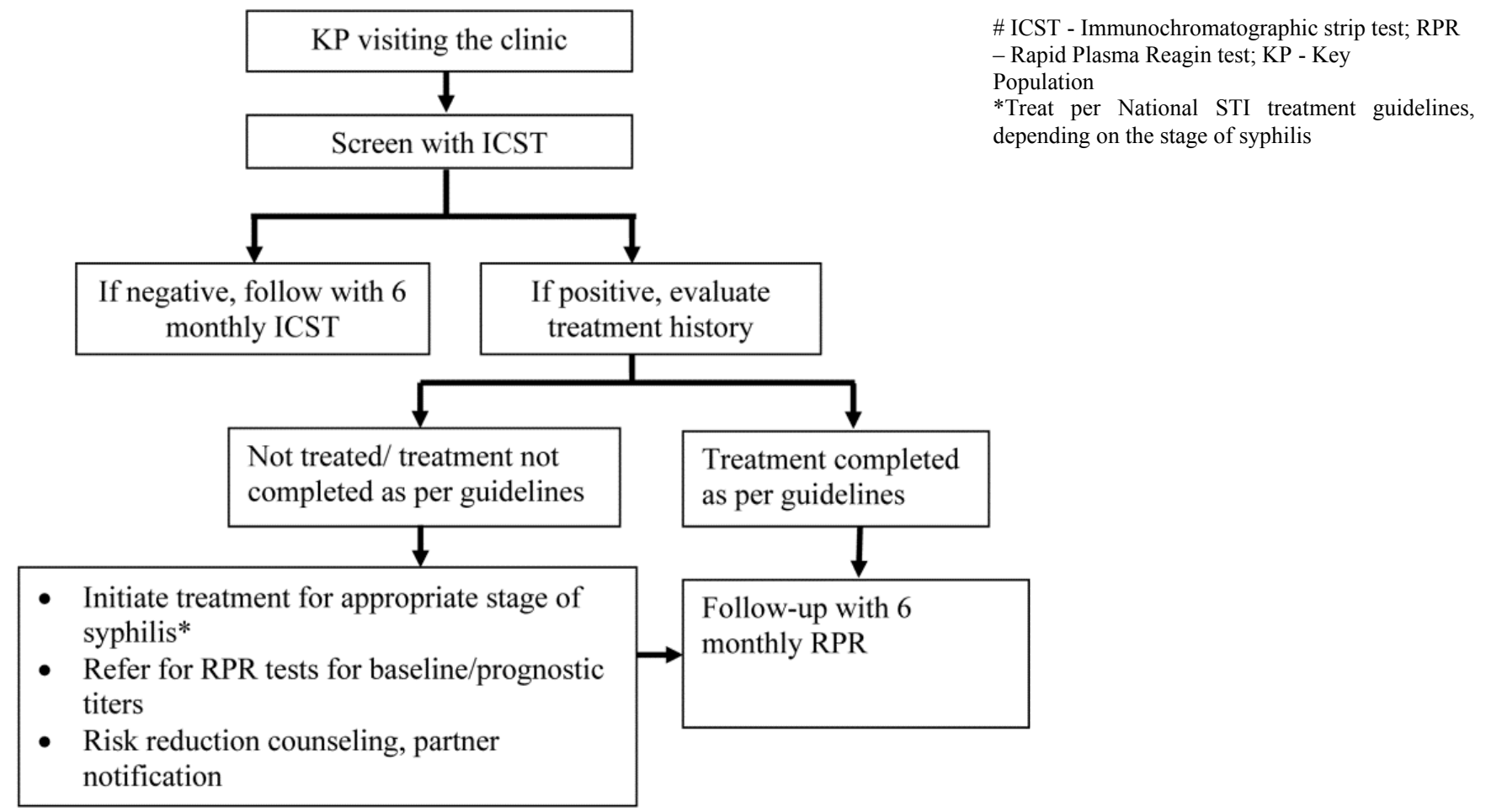

\# ICST - Immunochromatographic strip test; RPR - Rapid Plasma Reagin test; KP - Key

*Treat per National STI treatment guidelines, depending on the stage of syphilis 
an individual tracking database. The syphilis screening data, recorded in a separate register, was merged with the individual database using the unique identifiers. The individual tracking data was analysed using STATA-10 (StataCorp, College Station, Texas, US).

\section{Results}

The individual tracking database had a total of 2.65 million clinic visit records made by 330,000 female sex workers, 82,000 men who have sex with men, and 10,000 injecting drug users. The overall proportion of clinic attendees screened annually for syphilis increased consistently from $2.6 \%$ in 2005 to $21.6 \%$ in 2009 ( $\mathrm{p}<0.001)$ (Table). The increasing trend in coverage of syphilis screening was similar in all subgroups. From 2005 to 2009, the proportion of FSWs, MSM and IDUs screened for syphilis increased from $2.3 \%$ to $20.7 \%, 3.9 \%$ to $23.6 \%$ and $0.8 \%$ to $25.9 \%$ respectively.

The proportion of ICST used for screening increased from $7.4 \%$ in 2007 to $77.0 \%$ ( $p<0.001)$ in 2009 , replacing RPR as the predominant screening test. During the same period, the syphilis screening rates among clinic attendees increased from $9.0 \%$ to $21.6 \%(\mathrm{p}<0.001)$ (Table). A declining trend was found in the RPR reactivity rates, from $6.6 \%$ in 2006 to $4.4 \%$ in 2009 .

\section{Discussion}

The results showed that syphilis screening rates among Avahan clinic attendees had improved significantly from 2007 to 2009 . The introduction of ICST may also have contributed to this improvement. A study from Avahan sites at Mumbai and Thane also reported an increase in the uptake of syphilis testing among clinic attendees after introduction of the rapid tests. The screening coverage increased to $63.1 \%$, as compared to an earlier average of $14.3 \%$ by on-site RPR testing [7].

Sabidó et al. concluded that the performance of the point-of-care test combined with the advantages of onsite testing and same-day treatment were likely to improve coverage of syphilis screening in hard-toreach populations [8]. Though the sensitivity and specificity of rapid tests is less with whole blood when compared to serum, Siedner et al. suggest that it could be useful for the diagnosis of syphilis in field settings owing to its low cost, ease of use, and minimization of losses in follow-up [9]. The point-of-care test can also increase the likelihood of appropriate syphilis treatment. A study at Bangalore, India, showed that the sex workers treated for active syphilis (with reactive RPR and positive Treponema pallidum haemagglutination assay) after a positive on-site pointof-care test was $68.3 \%$, as compared to $44.8 \%$ in the control group whose serum samples were collected for off-site RPR testing [10].

The declining trend in syphilis sero-reactivity was possibly a result of the package of HIV/STI prevention interventions including condom promotion, presumptive treatment (including Azithromycin) and STI syndromic management. The Avahan evaluation study, the Integrated Behavioural and Biological Assessment, also showed an overall decline in syphilis prevalence from round-1(2005-07) to round-2 (20092010) among female sex workers across districts, ranging from $2.1 \%$ to $51.0 \%$ in 2009 and from $0.4 \%$ to $17.9 \%$ in 2010 [11].

In the Avahan program, wherever feasible, ICSTpositive clients were encouraged to have off-site RPR testing to decide on further management and followup. Syphilis management based on ICST alone (being a treponemal test) may result in overtreatment. However, syphilis management for key populations

Table. Uptake of syphilis screening at Avahan clinics, 2005-2009

\begin{tabular}{|c|r|r|r|r|r|r|}
\hline Year & $\begin{array}{c}\text { Total no. } \\
\text { of clinic } \\
\text { attendees }\end{array}$ & $\begin{array}{c}\text { No. of clinic } \\
\text { attendees } \\
\text { screened for } \\
\text { syphilis }\end{array}$ & $\begin{array}{c}\text { Proportion of } \\
\text { clinic attendees } \\
\text { screened }(\%)\end{array}$ & $\begin{array}{c}\text { Total tests } \\
\text { using ICST* }\end{array}$ & $\begin{array}{c}\text { Total tests } \\
\text { using } \\
\text { RPR* }\end{array}$ & $\begin{array}{c}\text { Proportion of } \\
\text { screening with } \\
\text { ICST (\%) }\end{array}$ \\
\hline 2005 & 43,394 & 1,115 & 2.6 & 0 & 1,768 & 0.0 \\
\hline 2006 & 108,836 & 5,925 & 5.4 & 0 & 9,209 & 0.0 \\
\hline 2007 & 169,612 & 15,280 & 9.0 & 1,362 & 17,168 & 7.4 \\
\hline 2008 & 220,876 & 29,311 & 13.3 & 16,717 & 17,108 & 49.4 \\
\hline 2009 & 286,990 & 61,897 & 21.6 & 54,424 & 16,250 & 77.0 \\
\hline
\end{tabular}

* Some individuals were screened for syphilis more than once in a year 
requires an operationally feasible strategy, ideally a simple test that can be performed in field conditions and with results available in a short time so that treatment can be initiated at the same visit. The hardto-reach nature of the key populations, low rates of follow-up visits, and higher risk of acquiring syphilis are likely to favour rapid tests in spite of the possibility of overtreatment. Montoya et al. suggest that in settings of high HIV prevalence and poor availability of syphilis diagnosis and treatment, patients who are TPHA-positive/RPR-negative would benefit from treatment [12].

Despite the efforts of the program, coverage of syphilis screening was sub-optimal. The relatively lower coverage (maximum of $21.6 \%$ ) shown by the individual tracking system could be due to some limitations. There was under-reporting of syphilis testing data (particularly when done through a camp approach) and some data without proper unique identification information was lost during the merging process. The design and protocol for individual tracking systems should be standardized at the outset with regular analysis for program monitoring. Moreover, incorporating the syphilis screening data in the clinic records and data quality checks at the clinic level would minimise data loss.

\section{Conclusion}

In summary, the Avahan data showed a consistent improvement in syphilis screening among clinic attendees over time with increasing use of ICST (over RPR) for initial screening and a declining trend in sero-reactivity. The Avahan experience shows that with systematic implementation of operationally feasible strategies, periodic syphilis screening can be effectively integrated into large-scale HIV prevention programs. The point-of-care rapid tests using fingerprick whole blood may have an impact on the improved coverage of syphilis screening. The pointof-care test thus has the potential of being scaled up to other resource-constrained primary care settings such as ante-natal clinics and other key population interventions. Periodic screening and appropriate treatment of syphilis, in conjunction with other HIV prevention measures (including STI management, presumptive treatment, risk reduction counselling and condom distribution), could contribute to an overall reduction in syphilis sero-reactivity among key populations over time.

\section{Acknowledgements}

Avahan is funded by the Bill and Melinda Gates Foundation. The views expressed herein are those of authors and do not necessarily reflect the official policy or position of the Bill and Melinda Gates Foundation.

\section{References}

1. Fleming DT, Wasserheit JN (1999) From epidemiological synergy to public health policy and practice: The contribution of other sexually transmitted diseases to sexual transmission of HIV. Sex Transm Infect 75: 2-17.

2. National AIDS Control Organization, India (2009) Report on Mid-Term Review of Sexually Transmitted Infection Services. Available: http://nacoonline.org/upload/STI\%20RTI\%20services/STI\%2 0RTI\%20MONOGRAPH\%20_NACP-III-.pdf. Accessed 28 February 2012.

3. Indian Council of Medical Research \& Family Health International, New Delhi. National Summary Report (December 2009), India Integrated Behavioral and Biological Assessment (IBBA), Round 1 (2005-2007). Available: http://www.fhi360.org/NR/rdonlyres/ewxhge5nvpq2i4mjuo7v g2vnlzizq6rge7npe46jwrr2fwpjqp2vdv6lazazuj2oe7lundg7gx thaf/IndiaNSRIBBAfn1010710.pdf. Accessed 28 February 2012.

4. Bill \& Melinda Gates Foundation, New Delhi, India (2008) Use It or Lose It: How Avahan Used Data to Shape its HIV Prevention Efforts in India. Available: http://www.gatesfoundation.org/avahan/Documents/Avahan_ UseItOrLooseIt.pdf. Accessed 28 February 2012.

5. Bill \& Melinda Gates Foundation, New Delhi, India (2009) Avahan-The India AIDS Initiative: FACT SHEET, December. Available: http://www.gatesfoundation.org/avahan/Documents/Avahan FactSheet.pdf. Accessed 28 February 2012.

6. Bill \& Melinda Gates Foundation, New Delhi, India (2010) Treat and Prevent: Avahan's Experience in Scaling Up STI Services to Groups at High Risk of HIV Infection in India. Available:

http://www.gatesfoundation.org/avahan/Documents/treat-andprevent.pdf. Accessed 28 February 2012.

7. Gupte S, Daly C, Agarwal V, Gaikwad SB, George B (2011) Introduction of rapid tests for large-scale syphilis screening among female, male, and transgender sex workers in Mumbai, India. Sex Transm Dis 38: 499-502.

8. Sabidó M, Benzaken AS, de-Andrade-Rodrigues EJ, Mayaud P (2009) Rapid point-of-care diagnostic test for syphilis in high-risk populations, Manaus, Brazil. Emerg Infect Dis 15: 647-649.

9. Siedner M, Zapitz V, Ishida M, De La Roca R, Klausner JD (2004) Performance of rapid syphilis tests in venous and fingerstick whole blood specimens. Sex Transm Dis 31: 557560.

10. Mishra S, Naik B, Venugopal B, Kudur P, Washington R, Becker M, John Kenneth, Krishnamurthy Jayanna, Banadakoppa M Ramesh, Shajy Isac, Marie-Claude Boily, James F Blanchard, Stephen Moses (2010) Syphilis screening among female sex workers in Bangalore, India: comparison of point-of-care testing and traditional serological approaches. Sex Transm Infect 86: 193-198. 
11. Indian Council of Medical Research and FHI 360, India (2011) National Summary Report - India, Integrated Behavioural and Biological Assessment (IBBA), Round 2 (2009-2010). Available: http://www.nariicmr.res.in/IBBA/121IBBA_Round_2_NSR.pdf. Accessed 28 February 2012.

12. Montoya PJ, Lukehart SA, Brentlinger PE, Blanco AJ, Floriano F, Sairosse J, Stephen Glyod (2006) Comparison of the diagnostic accuracy of a rapid immunochromatographic test and the rapid plasma reagin test for antenatal syphilis screening in Mozambique. Bull World Health Organ 84: 97104.

\section{Corresponding author}

Mugundu R. Parthasarathy

Technical Manager

STI Capacity Raising team

FHI360

H-5, Green Park Extension

New Delhi

India

Telephone: $+91-011$ - 40487777

Fax: +91-11-26172646

Email: pramien@fhi360.org

Conflict of interests: No conflict of interests is declared. 